\title{
Pattern Recognition Algorithm Based on Closeness Degree of Triangle Fuzzy Number
}

\author{
Yu-e Bao* and Erdun Bai \\ Inner Mongolia University of Nationalities, Inner Mongolia Tongliao, 028043, China \\ ${ }^{*}$ Corresponding author
}

\begin{abstract}
In this paper, for determining recognition target class problem in inaccurate or imprecise environments, we designed triangular fuzzy number pattern recognition algorithm based on closeness degree. Firstly, the attribute values of each standard test set and the sample set are converted into triangular fuzzy numbers. Secondly, the $E W$-type closeness formula is used to calculate closeness degree between the attributes of each standard set and sample set, and establish the relative closeness degree matrix. Thirdly, the target identification of class model is determined based on the relative closeness degree matrix. By identifying 20 kinds of perfume test data sets with the tag to verify the validity and rationality of the proposed algorithm.
\end{abstract}

Keywords-EW-type closeness degree; triangular fuzzy numbers; relative approach degree; pattern recognition

\section{INTRODUCTION}

Pattern recognition can be understood as a process of identification of the input data. The purpose of fuzzy pattern recognition system is assigning each input data to a possible model class [1-4]. The different input values with similar characteristics can be grouped into the same category, enter a value different characteristics into different categories. Fuzzy pattern recognition can be divided into two types: fuzzy pattern recognition direct method (maximum membership principle) and fuzzy pattern recognition indirect method (degree of closeness close principle). Indirect fuzzy pattern recognition is to investigate a fuzzy set and fuzzy sets which are known's close degree are the best.

In Ref. [5], it uses closeness theory of fuzzy sets and interval number to study the closeness degree of fuzzy numbers, and gives type integral similarity measures can reasonably effectively reflect the degree of two fuzzy numbers close. Triangular fuzzy number is a special kind of fuzzy numbers, has good usability when used to describe the lack of data, data accuracy is low, etc [6-8]. We use type integral closeness degree of fuzzy numbers to discussion on the triangular fuzzy number pattern recognition algorithm. Establish a pattern recognition algorithm based on type triangle fuzzy numbers close degree. And as an application of algorithm identify 20 kinds of perfume test data set with labels.

\section{PREREQUISITE}

Let $R$ be the real numbers field. A fuzzy set

$$
u: R \rightarrow[0,1]
$$

is called a fuzzy number if it is normal, convex, and upper semi-continuous and support set is a compact set. Let $\mathrm{F}_{0}$ be the set of all fuzzy numbers, and is called fuzzy number space. In particular, for $u \in \mathrm{F}_{0}$, if

$$
u(x)=\left\{\begin{array}{c}
\frac{x-a}{b-a}, a \leq x \leq b \\
\frac{c-x}{c-b}, b<x \leq c \\
0, \text { else }
\end{array}\right.
$$

then $u$ is called the triangular fuzzy numbers, and denoted as

$$
u=\langle a, b, c>
$$

For $u \in \mathrm{F}_{0}$, the $r$ - level set of $u$ :

$$
[u]^{r}=\left\{\begin{array}{c}
\{x \in R: u(x) \geq r\}, 0<r \leq 1, \\
\operatorname{supp} u, r=0 .
\end{array}\right.
$$

is a nonempty bounded closed interval on the $R$, which is denoted as

$$
[u]^{r}=[\underline{u}(r), \bar{u}(r)]
$$

And

$$
E\left([u]^{r}\right)=\frac{\underline{u}(r)+\bar{u}(r)}{2}
$$

$$
w\left([u]^{r}\right)=\frac{\bar{u}(r)-\underline{u}(r)}{2}
$$


are respectively the expectation and width of bounded closed interval $[u]^{r}$.

Theorem 1.1 ${ }^{[5]}$ Let mapping $N_{E W}^{p}: \mathrm{F}_{0} \times \mathrm{F}_{0} \rightarrow[0,1]$ defined as

$$
N_{E W}^{P}(u, v)=\left(\int_{0}^{1}\left(n_{E W}\left([u]^{r},[v]^{r}\right)\right)^{p} d r\right)^{\frac{1}{p}}(p \geq 1)
$$

Where $u, v \in \mathrm{F}_{0}$ and

$$
\begin{aligned}
& n_{E W}\left([u]^{r},[v]^{r}\right) \\
& \left.1+\left(|| E\left([u]^{r}\right)-\left.E\left([v]^{r}\right)\right|^{2}+\frac{1}{3}\left|W\left([u]^{r}\right)-W\left([v]^{r}\right)\right|^{2}\right)^{\frac{1}{2}}\right)^{n}
\end{aligned}
$$

then

$$
N_{E W}^{P}(u, v)
$$

becomes closeness degree between fuzzy numbers $u$ and $v$, and the closeness degree is called as $E W$ - Type integral closeness degree.

\section{TRIANGUlar FUZZY NUMBER PATtERn RECOGNITION ALGORITHM}

\section{A. Problem Description}

In some consider inaccurate or imprecise environments, how to identify the target belongs to which one of $l$ class.

\section{B. Dataset Description}

For each standard test set

$$
C_{k}(k=1,2, \ldots, l)
$$

we randomly choose $n(k)$ samples from each standard test set. By observation and evaluation of a property, we obtain $c_{i}^{k}$ which is the observation of the ith sample in $k$ th standard test set. Data set can be obtained as follows:

$$
\begin{aligned}
& C_{1}:\left[c_{1}^{1}, c_{2}^{1}, c_{3}^{1}, \ldots, c_{n(k)}^{1}\right] \\
& C_{2}:\left[c_{1}^{2}, c_{2}^{2}, c_{3}^{2}, \ldots, c_{n(k)}^{2}\right] \\
& \ldots \ldots \\
& C_{l}:\left[c_{1}^{l}, c_{2}^{l}, c_{3}^{l}, \ldots, c_{n(k)}^{l}\right]
\end{aligned}
$$

For each test set

$$
T_{j}(j=1,2, \ldots, m)
$$

we randomly choose $\bar{n}(j)$ samples from each test set. By observation and evaluation of a property, we obtain $t_{i}^{j}$ which is the observation of the $i$ th of samples in $j$ th standard test set of standards. Data set can be obtained as follows:

$$
\begin{aligned}
& T_{1}:\left[t_{1}^{1}, t_{2}^{1}, t_{3}^{1}, \ldots, t_{\bar{n}(j)}^{1}\right] \\
& T_{2}:\left[t_{1}^{2}, t_{2}^{2}, t_{3}^{2}, \ldots, t_{\bar{n}(j)}^{2}\right] \\
& \ldots \ldots \\
& T_{m}:\left[t_{1}^{m}, t_{2}^{m}, t_{3}^{m}, \ldots, t_{\bar{n}(j)}^{m}\right]
\end{aligned}
$$

Next, a triangular fuzzy number pattern recognition algorithm based on the $E W$ - type integral close degree is given.

\section{Step 1.}

For each

$$
C_{k}(k=1,2, \ldots, l)
$$

calculate the mean and standard deviation by the following formulas:

$$
\mu^{k}=\frac{1}{n(k)} \sum_{i=1}^{n(k)} c_{i}^{k}(k=1,2, \ldots, l)
$$

$$
\sigma^{k}=\sqrt{\frac{1}{n(k)-1} \sum_{i=1}^{n(k)}\left(c_{i}^{k}-\mu^{k}\right)^{2}}(k=1,2, \ldots, l)
$$

and construct triangular fuzzy number

$$
u^{k}(k=1,2, \ldots, l)
$$

where

$$
u^{k}(x)=\left\{\begin{array}{c}
\frac{x-\left(\mu^{k}-2 \sigma^{k}\right)}{2 \sigma^{k}}, \mu^{k}-2 \sigma^{k} \leq x \leq \mu^{k} \\
\frac{x-\left(\mu^{k}+2 \sigma^{k}\right)}{2 \sigma^{k}}, \mu^{k}<x \leq \mu^{k}+2 \sigma^{k} \\
0, \text { else }
\end{array}\right.
$$


Step 2.

For each $T_{j}(j=1,2, \ldots, m)$, calculate the mean and standard deviation by the following formulas:

$$
\begin{gathered}
\bar{\mu}^{j}=\frac{1}{n(j)} \sum_{i=1}^{n(j)} t_{i}^{j}(j=1,2, \ldots, m) \\
\bar{\sigma}^{j}=\sqrt{\frac{1}{n(j)-1} \sum_{i=1}^{n(j)}\left(t_{i}^{j}-\bar{\mu}^{j}\right)^{2}}(j=1,2, \ldots, m)
\end{gathered}
$$

and construct triangular fuzzy number $\bar{u}^{j}(j=1,2, \ldots, m)$ :

$$
\bar{u}^{j}(x)=\left\{\begin{array}{c}
\frac{x-\left(\bar{\mu}^{j}-10 \bar{\sigma}^{j}\right)}{10 \bar{\sigma}^{j}}, \bar{\mu}^{j}-10 \bar{\sigma}^{j} \leq x \leq \bar{\mu}^{j} \\
\frac{x-\left(\bar{\mu}^{j}+10 \bar{\sigma}^{j}\right)}{10 \bar{\sigma}^{j}}, \bar{\mu}^{j}<x \leq \bar{\mu}^{j}+10 \bar{\sigma}^{j} \\
0, \text { else }
\end{array}\right.
$$

Step 3.

Calculate

$$
N_{E W}^{p}\left(\bar{u}^{j}, u^{j}\right)(k=1,2, \ldots, l)(j=1,2, \ldots, m)
$$

which is the closeness degree between $\bar{u}^{j}$ and $u^{k}$, and establish the closeness degree matrix $N_{m \times l}$. The element of $j$ th row and $k$ th column of $N_{m \times l}$ is

$$
\begin{aligned}
& N_{E W}^{p}\left(\bar{u}^{j}, u^{k}\right)=\left(\int_{0}^{1}\left(n_{E W}\left(\left[\bar{u}^{j}\right]^{r},\left[u^{k}\right]^{r}\right)\right)^{p} d r\right)^{\frac{1}{p}} \\
& (p \geq 1, k=1,2, \ldots, l, j=1,2, \ldots, m)
\end{aligned}
$$

\section{Step 4.}

Establish a relative closeness matrix $P_{m \times l}$. The element of $j$ th row and $k$ th column of $P_{m \times l}$ is

$$
p\left(\bar{u}^{j}, u^{j}\right)=\frac{N_{E W}^{p}\left(\bar{u}^{j}, u^{k}\right)}{\sum_{k=1}^{l} N_{E W}^{p}\left(\bar{u}^{j}, u^{k}\right)}
$$

which is the relative closeness degree for tags of $j$-test data equivalent to tags of $k$-standard data.
Step 5.

Analyzing tag test data, if

$$
p\left(\bar{u}^{j}, u^{k_{0}}\right)=\max _{k=1,2, \cdots, l}\left\{p\left(\bar{u}^{j}, u^{k}\right)\right\}
$$

then the tags of $j$-test data equivalent to tags of $k_{0}$-standard data.

\section{NUMERICAL EXPERIMENTS}

In this section, we will introduce the experimental process of triangular fuzzy number pattern recognition algorithm based on the type integral Close degree, and compare it with other pattern recognition algorithms, and show the rationality and effectiveness of the algorithms.

Datasets are Perfume Data Set data sets which is the publicly available data sets [9] published in the UCI website. Data set is provided to the site by Professors Yousif Al-Bastaki in Kingdom of Bahrain and Professor Bekir KARLIK in Konya Turkey. The data was obtained from 20 different perfumes by using a handheld odor meter (OMX-GR sensor). Second record data, a total of 28 seconds. It includes standard data sets and test data sets and each data set have its labels.

Next, we use the triangular fuzzy number pattern recognition algorithm based on the $E W$ - type integral proximity degree to recognize 20 kinds of perfume test data sets, and compare them with the original algorithm. The identification process is as follows:

Step 1.

Using the formulas (1), (2), (4), (5) to calculate the expectation and variance of standard data sets and test data sets with labels. See Table1.

TABLE I. THE EXPECTATION AND VARIANCE OF STANDARD DATA SETS AND TEST DATA SETS WITH LABELS

\begin{tabular}{|c|c|c|c|c|}
\hline & $\mu$ & $\sigma$ & $\bar{\mu}$ & $\bar{\sigma}$ \\
\hline 1 & 64.4674 & 0.0703 & 64.2389 & 0.2157 \\
\hline 2 & 60.7788 & 0.2111 & 61.2327 & 0.1808 \\
\hline 3 & 57.8271 & 0.1748 & 56.7201 & 0.5227 \\
\hline 4 & 71.8107 & 0.3596 & 71.8976 & 0.3042 \\
\hline 5 & 68.1160 & 0.1001 & 68.1934 & 0.0361 \\
\hline 6 & 70.2926 & 0.4163 & 70.0835 & 0.0356 \\
\hline 7 & 60.4489 & 0.2395 & 70.0835 & 0.0356 \\
\hline 8 & 68.8180 & 0.2275 & 68.3761 & 0.1945 \\
\hline 9 & 72.2070 & 0.2374 & 71.8021 & 0.2181 \\
\hline 10 & 73.9805 & 0.2556 & 72.4985 & 0.0730 \\
\hline 11 & 62.8561 & 0.1270 & 63.0347 & 0.7765 \\
\hline 12 & 46.0148 & 0.0000 & 47.0155 & 1.0381 \\
\hline 13 & 82.4257 & 0.0354 & 82.3579 & 0.0003 \\
\hline 14 & 79.4276 & 0.2540 & 79.9276 & 0.0688 \\
\hline 15 & 85.0560 & 0.0000 & 85.0556 & 0.0000 \\
\hline 16 & 71.6419 & 0.2381 & 72.2133 & 0.2487 \\
\hline 17 & 66.5734 & 0.2790 & 68.6455 & 3.7527 \\
\hline 18 & 64.1674 & 0.9844 & 62.0563 & 0.5925 \\
\hline 19 & 66.8483 & 0.2388 & 66.2218 & 0.0000 \\
\hline 20 & 65.6467 & 0.2596 & 66.2877 & 0.1279 \\
\hline
\end{tabular}


Step 2.

Using the formulas (3) and (6) respectively to convert the data in Table 1 into triangular fuzzy numbers, and obtain Table 2.

TABLE II. TRIANGULAR FUZZY NUMBER OF STANDARD DATA SETS AND TEST DATA SETS WITH LABELS

\begin{tabular}{|c|l|l|}
\hline & Standard data sets & test data sets \\
\hline 1 & $<63.7644,64.4674,65.1703>$ & $<62.0817,64.2389,66.3960>$ \\
\hline 2 & $<58.6681,60.7788,62.8895>$ & $<59.4244,61.2327,63.0410>$ \\
\hline 3 & $<56.0794,57.8271,59.5747>$ & $<51.4931,56.7201,61.9471>$ \\
\hline 4 & $<68.2145,71.8107,75.4070>$ & $<68.8552,71.8976,74.9401>$ \\
\hline 5 & $<67.1152,68.1160,69.1168>$ & $<67.8324,68.1934,68.5545>$ \\
\hline 6 & $<66.1336,70.2969,74.4603>$ & $<69.7277,70.0835,70.4393>$ \\
\hline 7 & $<58.0539,60.4489,62.8439>$ & $<69.7277,70.0835,70.4393>$ \\
\hline 8 & $<66.5433,68.8180,71.0928>$ & $<66.4312,68.3761,70.3210>$ \\
\hline 9 & $<69.8335,72.2070,74.5806>$ & $<69.6210,71.8021,73.9832>$ \\
\hline 10 & $<71.4248,73.9805,76.5363>$ & $<71.7685,72.4985,73.2285>$ \\
\hline 11 & $<61.5863,62.8561,64.1260>$ & $<55.2702,63.0347,70.7993>$ \\
\hline 12 & $<46.0148,46.0148,46.0148>$ & $<36.6348,47.0155,57.3962>$ \\
\hline
\end{tabular}

\begin{tabular}{|c|c|c|}
\hline 13 & $<82.0714,82.4257,82.7800>$ & $<82.3553,82.3579,82.3605>$ \\
\hline 14 & $<76.8879,79.4276,81.9673>$ & $<79.2397,79.9276,80.6154>$ \\
\hline 15 & $<85.0560,85.0560,85.0560>$ & $<85.0556,85.0556,85.0556>$ \\
\hline 16 & $<69.2609,71.6419,74.0228>$ & $<69.7265,72.2133,74.7001>$ \\
\hline 17 & $<63.7839,66.5734,69.3630>$ & $<31.1181,68.6455,106.1730>$ \\
\hline 18 & $<54.3235,64.1674,74.0112>$ & $<56.1310,62.0563,67.9816>$ \\
\hline 19 & $<64.4603,66.8483,69.2362>$ & $<66.2217,66.2218,66.2219>$ \\
\hline 20 & $<63.0504,65.6467,68.2431>$ & $<65.0091,66.2877,67.5662>$ \\
\hline
\end{tabular}

Step 3.

Using the formula (7) to calculate closeness degree and establish closeness degree matrix $N_{20 \times 20}$.

\section{Step 4.}

Using the formula (8) to calculate relative closeness degree and establish relative closeness degree matrix $P_{20 \times 20}$.

By the above formulas and the relative closeness degree matrix (9), the Recognition results is in Table 3.

TABLE III. PATTERN RECOGNITION RESULTS

\begin{tabular}{|l|l|l|l|l|l|l|l|l|l|l|l|l|l|l|l|l|l|l|l|}
\hline 1 & 2 & 3 & 4 & 5 & 6 & 6 & 8 & 9 & 10 & 11 & 12 & 13 & 14 & 15 & 16 & 17 & 18 & 19 & 20 \\
\hline 1 & 2 & 3 & 4 & 5 & 6 & 6 & 8 & 9 & 9 & 11 & 12 & 13 & 14 & 15 & 16 & 8 & 11 & 19 & 20 \\
\hline
\end{tabular}

\section{Step 5.}

The first line is the original tags of test data sets, and the second line is the tags obtained by our proposed algorithm. The comparison results of Recognition accuracy rate between the proposed algorithm and other four pattern recognition algorithms can be seen in Table 4 .

TABLE IV. COMPARISON OF RECOGNITION ACCURACY RATE

\begin{tabular}{|l|l|}
\hline Name & Accuracy \\
\hline $\begin{array}{l}\text { Pattern recognition algorithm } \\
\text { based on EW-type triangle fuzzy } \\
\text { numbers close degree }\end{array}$ & $85 \%$ \\
\hline Bayes Classifier & $70 \%$ \\
\hline Logistic regression & $70 \%$ \\
\hline K-NN & $65 \%$ \\
\hline C4.5 & $55 \%$ \\
\hline
\end{tabular}

\section{CONCLUSIONS}

The design of triangular fuzzy number pattern recognition algorithm based on $E W$ - type integral Close degree is based on data set of multiple measurements on each dimension of each sample, so the algorithm is better than other algorithms in data description.

\section{ACKNOWLEDGMENTS}

This work is supported by the National Science Fund of China (11461052)

\section{REFERENCES}

[1] Chen Wen, Zhang Qiang. The Partner Selection in Virtual Enterprises Based on Fuzzy Pattern Recognition. Fuzzy Systems and Mathematics. 2006, vo 2. pp.146-151.
[2] Zhang Cai-qing, Lin Ming, Qi Ruo-nan. Atmospheric Quality Assessment in Coal-fired Power Plants Based on Fuzzy Pattern Recognition. Proceedings of the CSEE. 2009, vo 29. pp.30-34

[3] Zhou Li-juan, Xiao Man-sheng, Wen Zhi-qiang. Fuzzy identification method of incomplete text image based on grid closeness degree. Computer Engineering and Desigh. 2013, vo 12. pp. 4326-4329

[4] Shyi-Ming Chen, Shou-Hsiung Cheng, Tzu-Chun Lan. A novel similarity measure between intuitionistic fuzzy sets based on the centroid point so ftrans formed fuzzy numbers with applications to pattern cognition. Information Sciences. 2016, vo 343. pp.15-40

[5] Lei Yiming, Dai Bing , Bao Yu-e. The research of degree of closeness about fuzzy numbers. Pure and Applied Mathematics. 2016, vo 31. pp.611-619(in Chinese)

[6] Liu Sen, Zhao Xinan. Feature Recognition Based on individual advantage triangular fuzzy numbers. Statistics and Decision. 2014, Vo 21. pp.22-25. (in Chinese)

[7] Liu Xiu-mei, Zhao Ke-qin, Wang Chuan-bin. New multiple attribute decision-making model with triangular fuzzy numbers based on connection numbers. Systems Engineering and Electronics. 2009, vo10. pp.2399-2403. (in Chinese)

[8] Yu Chun-hai, Fan Zhi-ping. A Clustering Method for Multiple Attribute Information with Triangular Fuzzy Numbers Systems. Engineering Theory Methodology Applications. 2004, v05. pp. 467-470

[9] http://archive.ics.uci.edu/ml/datasets/Perfume+Data 